Service social

\title{
Le world café comme outil de mobilisation pour la sensibilisation à la maltraitance envers les proches aidants à Québec
}

\section{Sophie Éthier et Anne-Sophie Côté}

Volume 64, numéro 1, 2018

URI : https://id.erudit.org/iderudit/1055891ar

DOI : https://doi.org/10.7202/1055891ar

Aller au sommaire du numéro

Éditeur(s)

École de service social de l’Université Laval

ISSN

1708-1734 (numérique)

Découvrir la revue

Citer cet article

Éthier, S. \& Côté, A.-S. (2018). Le world café comme outil de mobilisation pour la sensibilisation à la maltraitance envers les proches aidants à Québec. Service social, 64(1), 65-78. https://doi.org/10.7202/1055891ar
Résumé de l'article

La provenance de la maltraitance envers les aînés est le plus souvent attribuée à leur entourage et expliquée par le stress de l'aidant ou les relations antérieures conflictuelles ou abusives. Cet article vise à mettre en lumière une problématique sociale méconnue : la maltraitance envers les proches aidants. Nous exposons la démarche et les résultats d'un " world café » organisé à Québec pour en discuter. Cet exercice de consultation collective suscite quelques pistes de réflexion. D’abord, l'impossibilité de dissocier la maltraitance envers les aidants de celle des aînés, tout en soulignant l'importance de lui réserver un traitement différencié. Aussi, l'absence d'évaluation de leurs besoins par les intervenants, dont les travailleurs sociaux, ainsi que le manque de reconnaissance et de soutien des aidants de la part de l'État comme facteurs de développement d'un terreau fertile à la maltraitance. 


\title{
Le world café comme outil de mobilisation pour la sensibilisation à la maltraitance envers les proches aidants à Québec
}

\author{
ÉTHIER, Sophie, Ph.D, gérontologie \\ Professeure agrégée à l'École de travail social et de criminologie \\ Université Laval \\ CÔTÉ, Anne-Sophie, T.S., M. Serv. Soc. \\ Deuil-Jeunesse
}

\section{RÉSUMÉ}

La provenance de la maltraitance envers les aînés est le plus souvent attribuée à leur entourage et expliquée par le stress de l'aidant ou les relations antérieures conflictuelles ou abusives. Cet article vise à mettre en lumière une problématique sociale méconnue : la maltraitance envers les proches aidants. Nous exposons la démarche et les résultats d'un "world café » organisé à Québec pour en discuter. Cet exercice de consultation collective suscite quelques pistes de réflexion. D'abord, l'impossibilité de dissocier la maltraitance envers les aidants de celle des aînés, tout en soulignant l'importance de lui réserver un traitement différencié. Aussi, l'absence d'évaluation de leurs besoins par les intervenants, dont les travailleurs sociaux, ainsi que le manque de reconnaissance et de soutien des aidants de la part de l'État comme facteurs de développement d'un terreau fertile à la maltraitance.

Mots-clés : maltraitance, proche aidance, world café.

\begin{abstract}
ABTRACT
The source of elderly mistreatment is most often attributed to those around them and may be explained by caregiver stress or previous conflictual or abusive relationships. This article will attempt to shed light on a little-known social problem: mistreatment towards caregivers. We will define and discuss the process and results of a world café organized in Quebec. This collective consultation exercise generates several reflections. First, the impossibility of dissociating elder mistreatment from caregivers mistreatment, while emphasizing the importance of differential treatment. Also, the lack of assessment of their needs by stakeholders, including social workers, as well as the lack of recognition and support of caregivers by the government as factors in the development of a fertile breeding ground for abuse.
\end{abstract}

Keywords: mistreatment, caregivers, world café. 


\section{INTRODUCTION}

Bien que décrite pour la première fois dans les écrits scientifiques britanniques dans les années 1970 (Baker, 1975 ; Burston, 1975), ce n'est que dans les années 1990 que la maltraitance envers les aînés devient l'objet d'une reconnaissance politique au Québec (Ministère de la Santé et des Services sociaux [MSSS], 1989, 1991), plus particulièrement depuis la mise en place du Plan d'action gouvernemental pour contrer la maltraitance envers les personnes aînées 2010-2015 (Beaulieu, 2012). S'appuyant sur la définition de la maltraitance de l'Organisation mondiale de la santé (OMS) élaborée en 2002 lors de la Déclaration de Toronto sur la prévention globale des mauvais traitements envers les aînés, le gouvernement québécois définit la maltraitance comme : « un geste singulier ou répétitif, ou une absence d'action appropriée, intentionnel ou non, [qui] se produit dans une relation où il devrait y avoir de la confiance, et que cela cause du tort ou de la détresse chez une personne aînée » (Ministère de la Famille, 2017, p. 15).

En raison du critère du lien de confiance nécessaire pour parler de maltraitance, celle-ci est presque exclusivement envisagée de manière unidirectionnelle (soit de la part de l'aidant envers l'aidé) et expliquée notamment par le stress de l'aidant (Killick, Taylor, Begley, Carter Anand et O'Brien, 2015 ; Roberto, McCann et Brossoie, 2013) ou par des relations antérieures difficiles et abusives (Kong et Moorman, 2015 ; Pickering, Mentes, Moon, Pieters et Phillips, 2015). Donc, selon la littérature, la maltraitance envers les personnes âgées provient principalement du proche aidant. Or, peu d'études se sont intéressées à la maltraitance envers les proches aidants alors que $29 \%$ d'entre eux seraient victimes de maltraitance au cours de leur expérience d'accompagnement d'un proche (Phillips, Torres de Ardon et Solis Briones, 2000).

Cet article vise à mettre en lumière cette problématique sociale peu abordée. De fait, les études à ce sujet se font rares et les politiques et pratiques dans ce domaine sont absentes. Nous exposerons la démarche et les résultats du " world café " - une méthode créative utilisée en travail social - organisé à Québec pour en discuter. La notion de proche aidant sera d'abord définie, puis celle de world café. Les discussions émanant du world café seront par la suite exposées. Une réflexion sur les retombées de cet exercice de consultation collective viendra clore l'article en confirmant le rôle du travail social face à cette problématique en émergence.

\section{Qu'est-ce qu'un proche aidant ?}

La notion d'aidant suscite depuis longtemps un certain débat, de sorte qu'il persiste encore une absence de consensus sur un terme spécifique pour nommer cette expérience d'aide et de soin, puis sur une définition précise pour la décrire dans les écrits scientifiques. Des divergences dans la définition et le rôle de l'aidant émergent, notamment, selon qu'il se définit lui-même comme tel ou qu'il est désigné par la personne âgée ou encore par les professionnels (Membrado, Vézina, Andrieu et Goulet, 2005). En français, les termes « aidant naturel », « aidant familial », " aidant informel » sont remplacés de plus en plus par " proche aidant », notamment au Québec où le « naturel » de l'aide a été maintes fois remis en question, particulièrement dans une perspective féministe (Ducharme, 2006 ; Guberman, Maheu et Maillé, 1991 ; Saint-Charles et Martin, 2001). De fait, selon Rita Therrien (1989), " cette dénomination cantonne cet apport dans une division sexuelle des tâches qui normalise l'interpellation faite aux femmes » (citée par Saint-Charles et Martin, 2001, p. 230). 
La personne aidante est presque toujours identifiée comme un membre de la famille et surtout définie en fonction de ce qu'elle « fait », c'est-à-dire par rapport aux tâches instrumentales qu'elle accomplit au quotidien (Ducharme, 2012). À titre d'illustration, l'OMS utilise la définition de Brodaty et Green (2000) selon laquelle l'aidant est « un membre de la famille ou un ami qui aide quelqu'un dans les tâches quotidiennes nécessaires à une vie autonome " (traduction libre de Brodaty et Green, 2000 cités par OMS, 2002, p. 3). La loi manitobaine sur la reconnaissance de l'apport des proches aidants (Gouvernement du Manitoba, 2011) décrit aussi l'aidant par le type d'aide, mais y ajoute les caractéristiques de l'aidé : «personne qui, sans rémunération et d'une façon informelle, donne des soins personnels, apporte un appui ou fournit de l'aide à une autre personne dont la vie est difficile en raison : a) d'un handicap, b) d'une maladie, c) d'une blessure, d) de l'âge » (p. 2). Au Québec, c'est la définition du ministère de la Santé et des Services sociaux qui est le plus souvent retenue : "Toute personne de l'entourage qui apporte un soutien significatif, continu ou occasionnel, à titre non professionnel, à une personne ayant une incapacité est considérée comme un proche aidant. II peut s'agir d'un membre de la famille ou d'un ami »(MSSS, 2003, p. 6). Nous retiendrons cette dernière définition dans le cadre de ce texte, en considérant que l'incapacité dont il est question peut provenir d'un handicap, d'une maladie, d'une blessure ou de l'âge, et qu'en conséquence, le rôle d'aidant peut se dérouler sur une période allant de quelques jours à de nombreuses années.

\section{Qu'en est-il de la maltraitance envers les proches aidants ?}

En 2012, $28 \%$ des Canadiens âgés de plus de 15 ans ont déclaré avoir « fourni des soins à un proche ayant un problème de santé de longue durée, une incapacité ou des problèmes liés au vieillissement » au moins 2 heures par semaine, faisant un peu plus de 8 millions de proches aidants au Canada (Turcotte, 2013, p. 2). Selon des études datant de plusieurs années, entre $34 \%$ (Steinmetz, 1988) et $51 \%$ des proches aidants (Hamel et al., 1990) seraient agressés verbalement par l'aidé. La violence physique serait également présente dans des proportions variant entre 18 et 34 \% (Coyne, Reichman et Berbig, 1993 ; Hamel et al., 1990 ; Paveza et al., 2000 ; Steinmetz, 1988). Sept pour cent des 213 proches aidants d'une étude de Hamel et ses collaborateurs (1990) révélaient même des violences sexuelles à leur endroit. La violence est particulièrement présente envers les femmes proches aidantes (Phillips, 2008 ; Phillips, Torres de Ardon et Solis Briones, 2000).

La maltraitance envers les proches aidants peut être le fruit d'un rapport relationnel complexe et s'expliquer par différents facteurs (Ayres et Woodtli, 2001 ; Fulmer et al, 2005 ; Ozcan, Boyacioglu et Sertcelik, 2017). Premièrement, les problèmes cognitifs ou psychiatriques de l'aidé peuvent l'amener à poser des gestes malencontreux ou à utiliser un langage inapproprié envers son aidant (Avieli, Smelov et Band-Winterstein, 2015 ; Band-Winterstein, Smeloy et Avieli, 2014 ; Hsu, Huang et Tu, 2014 ; Labrum, Solomon et Bressi, 2015 ; VandeWeerd et Paveza, 2005). Deuxièmement, les frustrations de l'aidé dues à son état de dépendance, à la perte de pouvoir inhérente à la relation d'aide ou au vieillissement peuvent expliquer certains gestes également (Steinmetz, 1988). Des antécédents de violence dans les relations (conjugales ou parentales) peuvent également être à l'origine de la maltraitance des aidés envers les proches aidants (Hamel et al., 1990 ; Liu, Kong, Bangerter, Zarit et Almeida, 2018).

Traitant le plus souvent de ses causes sous l'angle de la violence conjugale ou familiale, les études sur la maltraitance envers les proches aidants ne permettent pas toujours de bien appréhender ce phénomène dans sa spécificité, soit le contexte du rapport aidant-aidé, ses conséquences sur cette 
relation particulière, ainsi que sur la santé mentale de l'aidant (Kong, 2018). Bien que connu dans la littérature, le problème de la maltraitance des proches aidants n'est pas abordé dans les politiques et les pratiques de sorte qu'aucune mesure de prévention, de repérage ou d'intervention n'existe au Québec pour protéger ou soutenir les proches aidants qui en sont victimes. C'est dans cette perspective qu'un world café sur le thème de la maltraitance envers les proches aidants a été organisé à Québec.

\section{Le world café comme outil de réflexion collective en service social}

La méthode du world café, inventée en Californie en 1995 par Juanita Brown et David Isaacs et désormais pratiquée partout dans le monde, repose sur l'hypothèse que la connaissance et la sagesse dont nous avons besoin sont déjà présentes et accessibles et que l'intelligence apparaît lorsque les gens se connectent de manière créative (Brown et Isaacs, 2005). Le world café, qui peut réunir un groupe de 12 à plus de 1000 personnes, peut être utilisé dans le cadre d'un événement ponctuel de plus ou moins grande envergure ou être l'objet d'une démarche continue intégrée aux activités d'un organisme. Sept principes sous-tendent la méthode: définir le contexte ; créer un espace accueillant; explorer les questions qui importent; encourager la contribution de chacun; associer les diverses perspectives (principe de pollinisation) ; écouter les interventions des uns et des autres pour se donner un aperçu global cohérent; et partager les découvertes collectives (Brown et Isaacs, 2005). Le world café constitue un excellent outil de collecte de données en recherche et un moyen d'intervention efficace en travail social. De fait, les sept principes du world café rejoignent notamment les valeurs d'empowerment, de pratique axée sur le client, de promotion de la créativité, de partage d'information et d'apprentissage collectif promues par le service social (Fouché et Light, 2011).

Concrètement, les participants sont répartis autour de tables de quatre ou cinq personnes, dont l'une occupe le rôle d'hôte de table. Des cartons ou nappes en papier sont déposés sur les tables ainsi que des crayons de diverses couleurs. Le world café se déroule par vague. Première vague : une question est lancée au grand groupe par un animateur et une discussion s'entame entre les participants réunis autour de la même table. Ces derniers notent ou dessinent leurs idées et font des liens avec celles des autres. Après 20 minutes, l'animateur invite les participants à se diriger vers des tables différentes, sauf l'hôte de table qui demeure assis à sa place. L'hôte de table accueille les nouvelles personnes, procède à un bref résumé de la discussion qui a eu lieu précédemment, en s'appuyant sur les notes et dessins laissés par les participants sur la table. La discussion se poursuit, leurs successeurs amenant de nouvelles idées. Une fois le temps écoulé, on change les cartons ou les nappes afin de reprendre la procédure pour une seconde vague, avec une deuxième question. Selon le temps disponible, le world café comporte une à trois vagues. Une plénière avec l'ensemble des participants, en grand groupe, permet de faire ressortir les points saillants des discussions de chacune des tables. Les hôtes de table sont généralement responsables de faire ce compte rendu (Brown et Isaacs, 2005 ; Fouché et Light, 2011 ; Slocum, 2006). Certaines limites de la méthode du world café doivent toutefois être relevées. D'abord, il peut être difficile de trouver des questions (ou des thèmes) qui comptent vraiment pour l'ensemble des participants, et ensuite, de réunir un nombre important de personnes au même endroit, au même moment. 


\section{Résultats de notre world café}

Le world café a regroupé 27 participants provenant de différents milieux : 7 proches aidants, 6 étudiants universitaires et 11 intervenants provenant d'organismes communautaires ou du CIUSSS (Centre intégré universitaire de santé et de services sociaux) de la Capitale-Nationale, deux représentants de ministères et un de la Chaire de recherche sur la maltraitance envers les personnes aînées. Les participants ont été séparés en cinq tables.

\section{Selon vous, en quoi consiste la maltraitance envers les proches aidants ?}

Pour répondre à cette question, les participants ont échangé sur les formes de maltraitance, ses conséquences pour le proche aidant et ses causes. Selon les participants, la maltraitance envers un proche aidant peut être physique ou financière ou encore prendre la forme de la négligence. Les participants ont aussi mentionné que l'absence d'accompagnement et de soutien des aidants dans leur rôle est une autre forme de maltraitance. La violence psychologique est toutefois celle qui a le plus retenu l'attention. Cette dernière est par ailleurs difficile à identifier, en raison de ses manifestations parfois insidieuses: chantage, manipulation, demandes exigeantes, manque de respect, de sorte que les participants se demandent si elle est intentionnelle ou non. Or, peu importe l'intention réelle de celui qui l'exerce, la maltraitance envers l'aidant laisse des traces. Les participants ont nommé plusieurs conséquences: la faible estime de soi, l'isolement, la honte, l'accumulation d'émotions et de colère, l'épuisement, la culpabilité. La maltraitance, qui peut être vécue comme un traumatisme, pourrait même conduire l'aidant à l'autonégligence (par le biais de l'oubli de soi), notamment en raison des nombreuses pertes qu'elle provoque sur le plan de l'identité, du sentiment de sa valeur personnelle, des aspirations et objectifs personnels. Les participants ont également identifié plusieurs causes à cette maltraitance envers les aidants. En effet, il serait possible que la maltraitance relève d'un manque d'informations des aidants sur ce qui en constitue, de sorte qu'ils n'en réalisent pas l'ampleur ou la présence, ce qui les amène à normaliser et justifier leur situation. À l'inverse, s'ils sont conscients d'en vivre, ils peuvent avoir peur de dénoncer ce qu'ils vivent. La maltraitance peut par ailleurs émerger de la situation de l'aidé lui-même, difficile ou mal vécue par lui - celui-ci poserait par exemple des gestes de maltraitance en raison de sa frustration devant sa perte d'autonomie -, ou être le résultat d'une histoire relationnelle antérieure problématique, parfois marquée par la violence.

\section{D’où (de qui) pourrait provenir cette maltraitance?}

Cette question d'enrichissement a permis de poursuivre la discussion de la première vague en élargissant la notion de maltraitance, et donc de mieux cerner la manière dont les participants l'envisagent. Alors que les formes et manifestations de la maltraitance énumérées plus haut laissaient croire qu'elle provenait principalement de l'aidé ou de la dynamique relationnelle aidant-aidé, les participants ont ajouté, lors de cet approfondissement, d'autres sources potentielles de maltraitance envers les aidants : la famille immédiate et élargie, les amis, les voisins, les intervenants, le système de santé (Centre intégré universitaire de santé et de services sociaux [CIUSSS]), les milieux de travail non conciliants, la pression sociale. En somme, toutes les personnes qui minimisent le rôle et la situation du proche aidant, dont l'aidant lui-même, sont interpellées comme étant susceptibles de contribuer au phénomène. 
Comment la sensibilisation à la maltraitance envers les proches aidants pourrait-elle se faire ? et Qui seraient les personnes ciblées par cette sensibilisation et comment les rejoindre?

Ces questions ont constitué la deuxième vague de discussion. Ici, les participants ont d'emblée soulevé le double défi de la sensibilisation auprès des proches aidants eux-mêmes : d'abord, il faut que ceux-ci se reconnaissent comme proches aidants, et ensuite, qu'ils admettent être victimes de maltraitance. Les hommes aidants, qui assument un rôle social nouveau pour eux, sont particulièrement ciblés en raison de leur sentiment d'incompétence à l'accomplir. Les nombreuses personnes qui gravitent autour du proche aidant et de l'aidé, soit les familles, les amis, l'entourage et les intervenants doivent impérativement être sensibilisés à cette problématique afin de pouvoir la reconnaître, soutenir le proche aidant et éventuellement la dénoncer. Mais aussi, doivent être sensibilisés : la population en général, les organismes communautaires, l'Appui pour les proches aidants d'aînés, les médecins, les décideurs, le Secrétariat aux aînés, les policiers communautaires et les employeurs des proches aidants, en somme, tout le monde ! Les participants ont nommé un grand nombre de moyens susceptibles de contribuer à la sensibilisation au sujet de la maltraitance envers les proches aidants.

Pour en faciliter la présentation, ces moyens ont été classés selon les niveaux d'intervention écosystémique utilisés en service social : micro, méso et macro. Au niveau micro, les interventions consistent à venir en aide aux individus, aux familles et aux petits groupes dans le but d'améliorer leur qualité de vie, leur fonctionnement social ou leurs relations sociales. Au niveau méso, l'intervention vise davantage la communauté et les organisations formelles. L'objectif de ces interventions au niveau méso consiste le plus souvent à faciliter des changements dans les structures, dans les buts et le fonctionnement des groupes ou des institutions et dans l'organisation des services. Au niveau macro, l'intervention s'intéresse aux communautés, aux institutions et aux systèmes sociaux plus largement dans une optique de développement ou de changement dans les politiques, les lois ou les structures organisationnelles et même les valeurs sociales. L'organisation communautaire, le développement local, l'éducation publique et l'action sociale en font partie. Dans une perspective écosystémique, ces trois niveaux d'intervention sont interreliés et influent les uns sur les autres.

Les moyens de sensibilisation à la maltraitance au niveau micro concernent surtout sa prévention : miser sur le dépistage précoce des cas de maltraitance en informant les proches aidants sur leur rôle, en les outillant pour le remplir et en les soutenant (notamment via des groupes de soutien) afin qu'ils se reconnaissent comme aidants, comprennent ce qu'est la maltraitance et demandent de l'aide au besoin.

Les moyens de sensibilisation au niveau méso consistent plutôt à faire reconnaître la problématique par les milieux de pratique. Par exemple, intégrer un questionnaire dans le dossier des usagers du CLSC afin de dépister la maltraitance envers les proches aidants; faire de l'enseignement sur la problématique auprès des médecins et des étudiants en médecine; proposer des conférences sur le sujet lors de la semaine nationale des proches aidants ; concevoir des pièces de théâtre dans des milieux communautaires, distribuer des dépliants, faire des ateliers. Mais aussi, utiliser les médias sociaux, voire même le porte-à-porte, afin de donner de l'information directe.

Quant au niveau macro, les participants ont proposé des actions de grande envergure. Premièrement, adopter une politique nationale en faveur des proches aidants qui reconnaîtrait leur statut. Deuxièmement, informer la population sur l'apport considérable du travail des proches aidants sur le 
plan économique et social, et, en conséquence, sur la nécessité de prendre soin d'eux. Troisièmement, faire une campagne publicitaire illustrant des situations concrètes de maltraitance envers les aidants (comme cela existe pour la maltraitance des aînés). Et, quatrièmement, élaborer un programme de sensibilisation pour favoriser la concertation des actions proposées. Ces stratégies discutées par les participants sont présentées au tableau 1.

Tableau 1 : Stratégies de sensibilisation à la maltraitance envers les aidants selon le niveau d'intervention

\begin{tabular}{|c|c|}
\hline $\begin{array}{c}\text { Niveaux } \\
\text { d'intervention }\end{array}$ & Stratégies discutées en world café \\
\hline Micro & $\begin{array}{l}\text { - Miser sur le dépistage précoce des cas de maltraitance : informer les } \\
\text { aidants sur leur rôle et les outiller et les soutenir pour qu'ils puissent } \\
\text { bien le remplir. } \\
\text { - Viser à ce qu'ils se reconnaissent comme proches aidants, } \\
\text { comprennent ce qu'est la maltraitance et demandent de l'aide au } \\
\text { besoin. }\end{array}$ \\
\hline Méso & $\begin{array}{l}\text { - Faire reconnaître la problématique par les milieux de pratique : } \\
\text { intégrer un questionnaire de dépistage de la maltraitance envers les } \\
\text { aidants dans le dossier des usagers du CLSC ; } \\
\text { - faire de l'enseignement sur la problématique auprès des médecins } \\
\text { et des étudiants en médecine; } \\
\circ \text { proposer des conférences sur le sujet lors de la semaine des } \\
\text { proches aidants; } \\
\circ \text { concevoir des pièces de théâtre dans des milieux communautaires } \\
\circ \text { distribuer des dépliants; } \\
\circ \text { faire des ateliers; } \\
\circ \text { utiliser les médias sociaux, voire même le porte-à-porte, afin de } \\
\text { donner de l'information directe. }\end{array}$ \\
\hline Macro & $\begin{array}{l}\text { - Adopter une politique de reconnaissance du statut de proche aidant. } \\
\text { - Informer la population sur l'apport considérable du travail des proches } \\
\text { aidants sur le plan économique et social, et, en conséquence, sur la } \\
\text { nécessité de prendre soin d'eux. } \\
\text { - Faire une campagne publicitaire montrant aux citoyens des } \\
\text { situations concrètes de maltraitance envers les aidants. } \\
\text { - Élaborer un programme de sensibilisation pour favoriser la concertation } \\
\text { des actions proposées. }\end{array}$ \\
\hline
\end{tabular}

\section{Discussion : ET LA SUITE ?}

Les questions soumises au world café ont fait émerger plusieurs pistes de réflexion. La première renvoie à l'impossibilité de dissocier la maltraitance envers les aidants de celle envers les aînés. 
D'une part, $41 \%$ des proches aidants d'aînés sont eux-mêmes âgés de plus de 55 ans et $16 \%$ d'entre eux ont plus de 65 ans (L'Appui pour les proches aidants d'aînés, 2016). D'autre part, la maltraitance envers les aînés et celle envers les aidants comportent des origines communes (individuelles, relationnelles, organisationnelles et systémiques), ainsi que des conséquences et des pistes de solution similaires. La maltraitance envers l'aidant peut provenir de l'aidé, de l'entourage, des intervenants, du système de santé ou des aidants eux-mêmes via l'autonégligence. Ceci permet de saisir ce phénomène de manière plus globale et rejoint ce que l'on sait déjà des sources de maltraitance des aînés en général (Gouvernement du Québec, 2016). Conséquemment, les pistes de sensibilisation à ce phénomène devront nécessairement viser différentes cibles. Ces pistes, que nous avons classées selon les niveaux d'intervention (micro, méso ou macro), recoupent celles proposées par l'OMS en matière de prévention de la maltraitance des aînés. Ces dernières sont par ailleurs classées selon le public cible : interventions universelles (informations qui s'adressent à l'ensemble de la population) ; interventions sélectives qui ciblent les individus à risque ou concernés (pour nous, ce serait les proches aidants, leur entourage, les intervenants qui les côtoient) ; et interventions dirigées qui visent directement les personnes victimes (pour nous, les proches aidants) (World Health Organization [WHO], 2011). À la lumière de ces résultats, on pourrait penser que la maltraitance envers les proches aidants présente indéniablement des caractéristiques similaires à celles de la maltraitance envers les aînés. Or, dans le prolongement du point de vue des participants, cette problématique mérite toutefois un traitement différentiel, lequel devrait s'inscrire dans la réflexion plus globale sur la proche aidance afin d'en saisir également les singularités et de mettre en place les mécanismes appropriés.

Ceci nous conduit vers la seconde piste de réflexion à laquelle le world café nous convie. L'État québécois reconnaît depuis longtemps l'apport inestimable des proches aidants dans le maintien à domicile des personnes en perte d'autonomie et la nécessité de leur accorder des périodes de répit pour les soutenir (Ministère des Affaires sociales [MAS], 1985 ; MSSS, 1994, 2003). Toutefois, c'est depuis 2009, avec la loi 6 sur le Fonds de soutien aux proches aidants (2009) et le rapport du comité d'experts en vue de l'élaboration d'un plan d'action pour la maladie d'Alzheimer (Bergman, 2009), que les proches aidants sont plus particulièrement devenus un centre d'intérêt dans les politiques. Le Plan d'action gouvernemental pour la solidarité et l'inclusion sociale 2010-2015 (Ministère de l'Emploi et de la Solidarité sociale [MESS], 2010) ainsi que le Plan d'action gouvernemental pour l'égalité entre les femmes et les hommes 2011-2015 (Ministère de la Culture, des Communications et de la Condition féminine [MCCCF], 2011) mentionnent également la volonté politique de soutenir les proches aidants par le biais de services adaptés à leurs besoins. En 2012, la politique Vieillir et vivre ensemble - Chez soi, dans sa communauté, au Québec (communément appelée la politique VVE) (Ministère de la Famille et des Aînés [MFA] et MSSS, 2012) a réitéré la place des aidants et leurs besoins spécifiques:

La reconnaissance des proches aidants comme contributeurs majeurs pour permettre aux personnes aînées de demeurer le plus longtemps possible à domicile et dans leur communauté souligne l'importance d'agir en matière de soutien aux organismes qui leur viennent en aide et de mettre en place des mesures de conciliation travail-famille (p. 30).

Or, dans la pratique, en dépit du fait que Ducharme et ses collègues aient conçu et validé un outil d'évaluation des besoins des proches aidants en 2010 nommé Entente sur le soutien aux proches aidants (ESPA) (Ducharme et al., 2010), les besoins des proches aidants ne sont pas encore 
véritablement aussi considérés qu'ils le devraient par les travailleurs sociaux, ce qui contribue au développement d'un terreau fertile pour la maltraitance. Les orientations de la politique VVE visant à favoriser la participation des aînés dans leur communauté et le maintien de leur santé dans un environnement sain et sécuritaire (MFA et MSSS, 2012) ne peuvent se concrétiser sans l'apport de leurs proches aidants, lesquels ont aussi le droit de vieillir en continuant de participer, d'être en santé et en sécurité dans leur communauté.

Ce qui nous amène à la troisième piste de réflexion. Les participants ont proposé d'adopter une politique reconnaissant le statut de proche aidant. D'autres y ont également pensé. En 2015, L'Appui pour les proches aidants d'aînés a constitué un comité consultatif afin de :

[...] recommander les publics et les objectifs prioritaires en termes de changement ou d'adoption de comportements envers les proches aidants d'aînés ainsi que des mesures ou des pratiques favorables qui symboliseraient et confirmeraient la notion de valorisation du rôle des proches aidants d'aînés. II devait aussi identifier des stratégies de valorisation du rôle des proches aidants et formuler des recommandations quant à leur mise en œuvre (p. 6).

Parmi les recommandations du comité, on souhaitait que «l'Assemblée nationale du Québec manifeste solennellement sa propre reconnaissance à l'égard des proches aidants et que le gouvernement du Québec adopte une approche interministérielle afin de s'assurer que l'ensemble des programmes et des services gouvernementaux prenne pleinement en compte les divers besoins des proches aidants » (p. 10). Le comité soulignait toutefois l'importance que « les activités de valorisation du rôle des proches aidants d'aînés évitent les pièges que sont l'idéalisation, la stigmatisation, la culpabilisation, la pression indue et l'instrumentalisation des proches aidants » (p. 8).

L'Institut de planification des soins (IPS) et le Regroupement des aidantes et aidants naturels de Montréal (RAANM) ont produit en 2015 un rapport conjoint intitulé Vers la reconnaissance d'un statut légal pour les proches aidants dans le but que leurs droits soient reconnus et respectés. Plus précisément, ce rapport affirme le droit du proche aidant au choix libre et éclairé de devenir proche aidant ; de déterminer lui-même l'étendue des tâches qu'il est prêt à accomplir, notamment quant aux soins de fin de vie ; d'être intégré à l'équipe de soins ; d'être informé de l'état de santé de l'aidé ; d'être traité à égalité et de ne pas subir de discrimination en raison de sa situation familiale.

Nous avons déposé un mémoire dans le cadre de l'élaboration du plan d'action 2018-2023 de la politique Vieillir et vivre ensemble - Chez soi, dans sa communauté, au Québec intitulé Au-delà de l'âge, reconnaître et soutenir tous les proches aidants (2017). Dans ce mémoire, nous remettons en question l'orientation âgiste du gouvernement du Québec, c'est-à-dire le fait qu'il limite son soutien aux proches aidants d'aînés. Ainsi, nous recommandons la mise en place d'une Stratégie nationale de soutien aux proches aidants (SNSPA) fondée sur la même approche innovante que la politique québécoise sur le vieillissement, à savoir un partenariat interministériel et intersectoriel et le développement durable des communautés par la mise en œuvre de solutions adaptées aux besoins de tous les proches aidants et répondant aux priorités du milieu. Pour ce faire, la SNSPA proposée comporte 10 leviers d'action, dont ceux d'offrir une reconnaissance juridique aux proches aidants, de permettre un développement durable des services communautaires leur étant destinés, de fournir des réponses adaptées à la diversité des proches aidants et d'élargir la gamme des mesures de soutien économique (Éthier, 2017). Par ailleurs, le premier principe qui devrait guider une telle stratégie est 
celui de l'engagement volontaire du proche aidant, tel qu'énoncé dans la politique sur le soutien à domicile en 2003.

Plus récemment, le Regroupement des aidants naturels du Québec (RANQ) a lancé sa propre Stratégie nationale de soutien aux proches aidants: Valoriser et épauler les proches aidants, ces alliés incontournables pour un Québec équitable (2018). Cette stratégie est fondée sur cinq principes : (1) le choix libre, éclairé et révocable, (2) la reconnaissance de l'expertise des proches aidants, (3) l'évaluation complète et annuelle des besoins des proches aidants, (4) la lutte contre l'appauvrissement des proches aidants et (5) le développement des connaissances dans le domaine. Reste à savoir si le gouvernement québécois prendra en compte ces stratégies complémentaires de valorisation et de reconnaissance du rôle et des droits des proches aidants afin de mieux les soutenir. La reconnaissance sociale du rôle fondamental que jouent les proches aidants constitue une part importante de notre responsabilité collective et une première étape essentielle à franchir.

Reconnaître qu'ils sont susceptibles de vivre de la maltraitance dans l'exercice de leur rôle en est une seconde. Le projet de loi 115 sanctionné le 30 mai 2017 par le gouvernement du Québec, intitulé Loi visant à lutter contre la maltraitance envers les aînés et toute autre personne majeure en situation de vulnérabilité, aurait pu constituer un espoir en ce sens. De fait, la loi oblige l'adoption d'une politique de lutte contre la maltraitance par tous les établissements de santé et de services sociaux et rend obligatoire le signalement de certains cas de maltraitance. Pour le gouvernement québécois, une personne en situation de vulnérabilité est : « une personne majeure dont la capacité de demander ou d'obtenir de l'aide est limitée temporairement ou de façon permanente, en raison notamment d'une contrainte, d'une maladie, d'une blessure ou d'un handicap, lesquels peuvent être d'ordre physique, cognitif ou psychologique » (Assemblée nationale, 2017, p. 6). Les proches aidants, s'ils reçoivent des services sociaux et de santé, pourraient ainsi être considérés comme des personnes en situation de vulnérabilité, ce qui rendrait obligatoire le signalement des formes de maltraitance qu'ils subissent. Or, comme ils ne sont pas spécifiquement ciblés dans la loi, peu d'intervenants les incluent dans cette définition, et peu de proches aidants pensent qu'elle peut s'appliquer à eux.

En somme, ce que l'on retient du world café, c'est que nous ne pouvons plus nous payer le luxe d'ignorer l'apport inestimable des proches aidants dans le maintien du système de santé et de solidarité sociale actuel, et encore moins de fermer les yeux sur la maltraitance qu'ils subissent dans l'accomplissement de ce rôle si fondamental et si utile à la société. Kempeneers, Battaglini et Van Pevenage (2015) montrent que l'épuisement des aidants peut provenir d'un manque de soutien de la part de l'État. Ce manque de soutien serait-il une forme de maltraitance envers les proches aidants ? La question demande une importante réflexion de la part des travailleurs sociaux. En tout cas, les participants au world café l'ont soulevée et y ont répondu par l'affirmative. Cela mérite qu'on s'y arrête un instant. La maltraitance envers les proches aidants existe. II nous semble impératif d'y voir dès maintenant et de faire rapidement des choix de société qui iront dans le sens de favoriser une culture de bientraitance à leur égard.

C'est aussi le souhait de la ministre des Aînés et des Proches aidants, Madame Marguerite Blais, qui a organisé une consultation en décembre 2018 dans le but de jeter les bases de ce qui deviendra la première politique nationale pour les proches aidants au Québec. La nécessité de le faire n'est plus à démontrer, mais cette fois la volonté politique est au rendez-vous! 


\section{RÉFÉRENCES}

Assemblée nationale (2017). Projet de loi $n^{\circ} 115$ : Loi visant à lutter contre la maltraitance envers les aînés et toute autre personne majeure en situation de vulnérabilité. (Présenté le 19 octobre 2016. Adopté le 30 mai 2017.)

Avieli, H., Smeloy, Y., et Band-Winterstein, T. (2015). Departure scripts and life review of parents living with abusive adult children with mental disorder. Journal of Aging Studies, 3448-56.

Ayres, M. M., et Woodtli, A. (2001). Concept analysis: Abuse of ageing caregivers by elderly care recipients. Journal of Advanced Nursing, 35(3), 326-334.

Baker, A. (1975). Granny battering. Modern Geriatrics, 5(8), 20-24.

Band-Winterstein, T., Smeloy, Y., et Avieli, H. (2014). Shared reality of the abusive and the vulnerable: the experience of aging for parents living with abusive adult children coping with mental disorder. International Psychogeriatrics, 26(11), 1917-1927.

Beaulieu, M. (2012). Contrer la maltraitance envers les personnes aînées au Québec : bilan historique des politiques publiques et inventaire des principales actions. Risques \& Qualité, IX(4), 59-65.

Bergman, H. (présidé par) (2009). Relever le défi de la maladie d'Alzheimer et des maladies apparentées. Une vision centrée sur la personne, l'humanisme et l'excellence. Rapport du comité d'experts en vue de l'élaboration d'un plan d'action pour la maladie d'Alzheimer. Québec, Canada : Gouvernement du Québec, Direction des communications du Ministère de la Santé et des Services sociaux du Québec.

Brown, J., Isaacs, D., et la World Café Community (CWC) (2005). The World Café Book: Shaping our futures through conversations that matter. San Francisco : Berett-Koehler Publishers.

Burston, G. W. (1975). Granny-battering. British Medical Journals, 3(6), 592.

Coyne, A. C., Reichman, W. E., et Berbig, L. J. (1993). The relationship between dementia and elder abuse. American Journal of Psychiatry, 150(4), 643-646.

Ducharme, F. (2006). Famille et soins aux personnes âgées. Enjeux, défis et stratégies. Montréal : Beauchemin et Chenelière Éducation.

Ducharme, F. (2012). Prendre soin d'un parent âgé vulnérable, une réflexion sur le concept de " proche aidant » et de ses attributs. Les Cahiers de l'année gérontologique, 4(2), 74-78.

Ducharme, F., Lévesque, L., Caron, C., Hanson, E., Magnusson, L., Nolan, J., et Nolan, M. (2010). Mise à l'essai d'un outil d'évaluation des besoins de soutien des proches-aidants d'un parent âgé à domicile : un outil ayant un potentiel d'application en Europe francophone. Recherche en soins infirmiers, 2(101), 67-80. 
Éthier, S. (2017). Au-delà de l'âge, reconnaître et soutenir tous les proches aidants. Mémoire présenté au Ministère de la Famille dans le cadre de l'élaboration du plan d'action 2018-2023 de la politique Vieillir et vivre ensemble - Chez soi, dans sa communauté, au Québec. Document inédit.

Fouché, C., et Light, G. (2011). An invitation to dialogue: The World Café in social work research. Qualitative Social Work, 10(1), 28-48.

Fulmer, T., Paveza, G., VandeWeerd, C., Fairchild, S., Guadagno, L., Bolton-Blatt, M., et Norman, R. (2005). Dyadic vulnerability and risk profiling for elder neglect. Gerontologist, 45(4), 525-534.

Gouvernement du Manitoba (2011). Loi sur la reconnaissance de l'apport des aidants naturels. C.C24.

Gouvernement du Québec (2016). Guide de référence pour contrer la maltraitance envers les personnes aînées. Gouvernement du Québec : Le Centre d'expertise en santé de Sherbrooke.

Guberman, N., Maheu, P., et Maillé, C. (1991). Et si l'amour ne suffisait pas... Femmes, familles et adultes dépendants. Montréal : Les Éditions du remue-ménage.

Hamel, M., Gold, D. P., Andres, D., Reis, M., Dastoor, D., Grauer, H., et Bergman, H. (1990). Predictors and consequences of aggressive behavior by community-based dementia patients. Gerontologist, 30(2), 206-211.

Hsu, M., Huang, C., et Tu, C. (2014). Violence and mood disorder : Views and experiences of adult patients with mood disorders using violence toward their parents. Perspectives in Psychiatric Care, 50(2), 111-121.

Institut de planification des soins (IPS) et Regroupement des aidantes et aidants naturels de Montréal (RAANM) (2015). Vers la reconnaissance d'un statut légal pour les proches aidants. Rapport conjoint. Disponible en ligne et à Bibliothèque et Archives nationales du Québec.

Kempeneers, M., Battaglini, A., et Van Pevenage, I. (2015). Chiffrer les solidarités familiales, Carnetsynthèse. Montréal : CIUSSS Nord-de-l'lle-de-Montréal.

Killick, C., Taylor, B. J., Begley, E., Carter Anand, J., et O'Brien, M. (2015). Older people's conceptualization of abuse: A systematic review. Journal of Elder Abuse \& Neglect, 27(2), 100-120.

Kong, J. (2018). Effect of caring for an abusive parent on mental health: The mediating role of selfesteem. Gerontologist, 58(3), 456-466

Kong, J., et Moorman, S. M. (2015). Caring for my abuser: Childhood maltreatment and caregiver depression. Gerontologist, 55(4), 656-666.

L'Appui pour les proches aidants d'aînés (2015). Rapport final - Comité consultatif de l'Appui national sur la valorisation du rôle des proches aidants d'aînés. 
Labrum, T., Solomon, P., et Bressi, S. K. (2015). Physical, financial, and psychological abuse committed against older women by relatives with psychiatric disorders: Extent of the problem. Journal of Elder Abuse \& Neglect, 27(4/5), 377-391.

Liu, Y., Kong, J., Bangerter, L. R., Zarit, S. H., et Almeida, D. M. (2018). Early parental abuse and daily assistance to aging parents with disability: Associations with the middle-aged adults' daily well-being. The Journals of Gerontology. Series B, Psychological Sciences and Social Sciences, 73(5), e59-e68.

Membrado, M., Vézina, J., Andrieu, S., et Goulet, V. (2005). Définitions de l'aide : des experts aux profanes. Dans S. Clément et J.-P. Lavoie (dir.), Prendre soin d'un proche âgé. Les enseignements de la France et du Québec. Ramonville-Saint-Agne : Éditions Érès.

Ministère de l'Emploi et de la Solidarité sociale (MESS) (2010). Le Québec mobilisé contre la pauvreté - Plan d'action gouvernemental pour la solidarité et l'inclusion sociale 2010-2015. Québec: Gouvernement du Québec.

Ministère de la Culture, des Communication et de la Condition féminine (MCCCF) (2011). Pour que l'égalité de droit devienne une égalité de fait. Plan d'action gouvernemetnal pour l'égalité entre les femmes et les hommes 2011-2015. Québec : MCCCF.

Ministère de la Famille - Secrétariat aux aînés (2017). Plan d'action gouvernemental pour contrer la maltraitance envers les personnes aînées 2017-2022. Québec : Gouvernement du Québec.

Ministère de la Famille et des Aînés (MFA) et Ministère de la Santé et des Services sociaux (MSSS) (2012). Vieillir et vivre ensemble - Chez soi, dans sa communauté, au Québec. Québec: Gouvernement du Québec.

Ministère de la Santé et des Services sociaux (MSSS) (1989). Vieillir... en toute liberté. Rapport du Comité sur les abus exercés à l'endroit des personnes âgées. Québec : Gouvernement du Québec.

Ministère de la Santé et des Services sociaux (MSSS) (1991). Vers un nouvel équilibre des âges. Rapport du Groupe d'experts sur les personnes âgées. Québec : Gouvernement du Québec.

Ministère de la Santé et des Services sociaux (MSSS) (1994). Les services à domicile de première ligne - Cadre de référence. Québec : Gouvernement du Québec.

Ministère de la Santé et des Services sociaux (MSSS) (2003). Chez soi : le premier choix. La politique de soutien à domicile. Québec : Gouvernement du Québec.

Ministère des Affaires sociales (MAS) (1985). Un nouvel âge à partager - Politique du ministère des Affaires sociales à l'égard des personnes âgées. Québec : Gouvernement du Québec.

Organisation mondiale de la santé (OMS) (2002). Current and future long-term care needs: An analysis based on the 1990 WHO study. The global burden of disease, and the international 
classification of functioning, disability and health. Disponible en ligne (consulté le 10 mai 2012) : http://www.who.int/chp/knowledge/publications/ltc_needs.pdf

Ozcan, N. K., Boyacioglu, N. E., et Sertcelik, E. (2017). Reciprocal abuse: Elder neglect and abuse by primary caregivers and caregiver burden and abuse in Turkey. Archives of Psychiatric Nursing, 31(2), 177-182.

Paveza, G.J., Cohen, D., Eisdorfer, C., Freels, S., Semia, T., Ashford, J., Gorelick, P.Philips, L.R., Torres de Ardon, E. et Solis Briones, G (2000). Abuse of female Caregivers by Care Recipients: Another Form of Elder Abuse. Journal of Elder \& Neglect, 12(3/4), 12-143.

Phillips, L. R., Torres de Ardon, E., et Solis Briones, G. (2000). Abuse of female caregivers by care recipients: Another form of elder abuse. Journal of Elder Abuse \& Neglect, 12(3/4), 123-143.

Phillips, L. R. (2008). Abuse of aging caregivers: Test of a nursing intervention. Advances in Nursing Science, 31(2), 164-181.

Pickering, C. E. Z., Mentes, J. C., Moon, A., Pieters, H., et Phillips, L. R. (2015). Adult daughters' descriptions of their mother-daughter relationship in the context of chronic conflict. Journal of Elder Abuse \& Neglect, 27(4/5), 356-376.

Regroupement des aidants naturels du Québec (RANQ) (2018). Valoriser et épauler les proches aidants, ces alliés incontournables pour un Québec équitable. Montréal.

Roberto, K. A., McCann, B. R., et Brossoie, N. (2013). Intimate partner violence in late life: An analysis of national news reports. Journal of Elder Abuse \& Neglect, 25(3), 230-241.

Saint-Charles, D., et Martin, J.-C. (2001). De la perspective d'« aidant naturel » à celle de «prochesoignant » : un passage nécessaire. Santé mentale au Québec, 26(2), 227-244.

Slocum, N. (2006). Méthodes participatives. Un guide pour l'utilisateur: le World Café. Bruxelles: Fondation Roi Baudoin.

Steinmetz, S. (1988). Duty bound: Elder abuse and family care. Beverly Hills : Sage Publications.

Turcotte, M. (2013). Etre aidant familial : quelles sont les conséquences? Regards sur la société canadienne. Publication $n^{0} 75-006-X$. Ottawa : Statistique Canada.

VandeWeerd, C., et Paveza, G. J. (2005). Verbal mistreatment in older adults: A look at persons with Alzheimer's disease and their caregivers in the state of Florida. Journal of Elder Abuse \& Neglect, $17(4), 11-30$.

World Health Organization (WHO) (2011). Interventions to prevent and reduce elder maltreatment (p. 43-57). Dans Dinesh Sethi et al., European report on preventing elder maltreatment. Danemark : WHO Regional Office for Europe. 